\title{
Experimental Characterisation of Catalyst-Free Carbon Nanomaterials from Mixed Vegetable and Animal Base Oils through Modified Traditional Process
}

\author{
P. S. Venkatanarayanan, ${ }^{1}$ R. Velmurugan, ${ }^{2}$ and A. Joseph Stanley ${ }^{1}$ \\ ${ }^{1}$ Madras Institute of Technology, Anna University, Chennai 600044, India \\ ${ }^{2}$ Aerospace Engineering, Indian Institute of Technology Madras, Chennai 600036, India
}

Correspondence should be addressed to P. S. Venkatanarayanan,psvenky_ravi@in.com

Received 26 May 2010; Accepted 10 March 2011

Academic Editor: Kin Tak Lau

Copyright () 2011 P. S. Venkatanarayanan et al. This is an open access article distributed under the Creative Commons Attribution License, which permits unrestricted use, distribution, and reproduction in any medium, provided the original work is properly cited.

\begin{abstract}
A new process for the preparation of catalyst-free pure carbon nanomatereials is described here. These nanomaterials are then experimentally investigated. The process is simple and traditional so that the operator can have full control over the flame geometry and oil consumption in a span of time. A slight modification is carried out in the traditional process with an option of external heating (forced convection). Two types of samples are prepared (S1 \& S2) from two different processes, Modified Traditional Process without external heating (MTP1) and with external heating (MTP2). Deep investigation on yield, morphology and structure of prepared samples are carried out by sophisticated analytical instruments. It is noted that the S1 sample contains pure carbon nano crystals along with well grown thin nano tubular structures whereas S2 sample contains pure carbon nanocrystals in a chain formation and likely possibility for the growth of nanotubes without any further treatment. It is observed clearly that the average weight gain of sample S2 is 22\% for MTP1 and 7\% for MTP2 more than that of the sample S1 over the same period of time. The range in dimension of the nanoparticles is varying from $6-45 \mathrm{~nm}$.
\end{abstract}

\section{Introduction}

The initial development of carbon nanomaterials through complicated and lengthy processes had been reported since 1970s. Several methods were used to develop the carbon nanomaterials. The morphology of soot particles was mentioned in [1]. The morphology shows the cluster of spheres. Each sphere is containing $10^{5}$ carbon atoms and $10^{4}-10^{5}$ hydrogen atoms. Carbon black (CB) improves the mechanical and electrical properties of high-performance materials. These particles give flexible filler network and strong polymer filler couplings [2]. The dynamic properties (rolling frictional properties) of carbon black can be improved by a simple process mentioned in [3]. Carbon black is a material composed essentially of elemental carbon in the form of quasispherical particles that are fused together into aggregates. It is obtained by partial combustion or thermal decomposition of hydrocarbons. Although several kinds of $\mathrm{CB}$ such as channel blacks, thermal blacks, or Lampblacks can be obtained through different processes [4]. ASTM D6556-10 Standard Test Method for Carbon Black to find the total surface area is referred. A variety of analytical techniques are used to identify the chemical groups present in the carbon surface [5]. A review is given on the surface chemistry of carbon blacks and other carbons, in particular, CBs. The main part is devoted to surface oxides with emphasis on the chemical methods used in the assessment and identification of surface functional groups. Reaction with free organic radicals can be used for the functionalization of carbon surfaces [6].

The Arc discharge method [7] was used in 1991 to prepare the carbon nanomaterials. These nanostructures contain one or more tubular walls with open or closed ends with geometrical disorders. The intensive study was carried out on carbon nanomaterials which were prepared out of amorphous carbon film. The graphitic structure, 
inherent to the production process of the amorphous carbon film, was investigated through electron microscope [8]. The aggregated nanomaterials were prepared when fuel-gas combustion was taking place and were investigated through analytical instruments [9]. Here natural gas, propane, and methane were combusted, and the aggregates were collected indoor and outdoor with lot of impurities.

Development of carbon nanomaterials by the catalyst influenced pyrolysis of phenol formaldehyde resin [10], and water soluble carbon nanotubes out of carbon soot extracted from burning of mustered oil in a lamp [11] were the significant derivatives with impurities and defects. Lowtemperature synthesis of carbon nanomaterials had been reviewed [12].

Much research attention had been paid to synthesis and characterisation of carbon nanomaterials particularly MWCNT and SWCNT. The pulsed laser ablation of carbon showed the particle surface profile with dimension of the order of $100 \mathrm{~nm}$ deposited on the substrate together with large amount of amorphous carbon phase [13].

Thermolysis method, a catalyst free process, was used to prepare the carbon nanomaterials in the presence of buffer gas. The carbon extract collected through this method contained carbon-onions, amorphous carbons, and impurities [14]. The carbon products developed through various costly and complicated methods contained impurities like industrial contaminants, catalytic impurities, and oxides. An exclusive and lengthy method for purifying the carbon products had also been reviewed [15].

The variation in size of the nanomaterials along with impurities due to the influence of catalyst had been cited [16]. The carbon products particularly fullerenes had been thoroughly investigated for their structural properties and applications [17]. The structure of carbon nanomaterials for cell dimension and phase with crystal planes had been studied [18]. Most of the properties of the nanomaterials depended on diameter and helicity of the atomic structure [19]. The importance of controlling the ambient parameters which influenced the size of the nanoparticles and structure was understood [20]. The carbon nanomaterials continue to gain the more importance for many potential applications because of their mechanical, electrical, and electronics properties.

Herein, we report a simple, modified Indian traditional and low-cost method to prepare the carbon nanomaterials from two different oil bases, that is, animal (S1) and mixed vegetable (S2). The developed method and products are free of the drawbacks discussed above. The modified traditional method involves the controlled combustion of [mixed vegetable base and animal base] oil in an earthen traditional lamp enclosed by combustion chamber which can be externally heated. The samples were made to deposit separately in a smooth ceramic/stainless steel substrate without any changes in the basic setup. A simple purification process is followed to purify the obtained samples. These two samples were investigated thoroughly for their yield, purity, geometry, and suitability for any specific potential applications through various sophisticated analytical instruments.

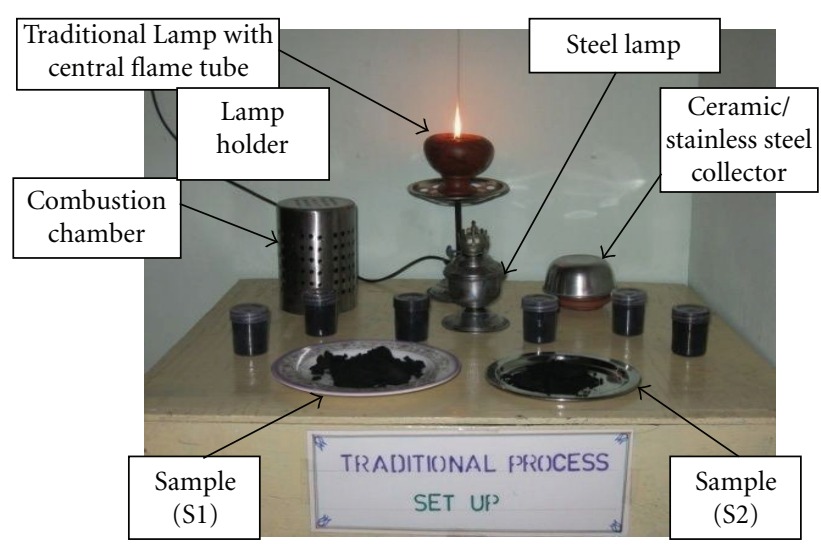

FIgUre 1: The modified traditional process (MTP) setup.

In olden days, the "big south Indian temples" were using vegetable oils like Mahua, punnai, neem, and gingili. for burning lamps to luminate the entire area during night time. The carbon deposits were noticed along the walls of the lamp enclosures and were used to be collected as carbon powders for medicinal purposes. The "Vedas, Puranas, and Ithikasas" are insisting the use of cow ghee and gingili for "yagas \& yagnas" to communicate with the divine power because of their sanctity and purity. Hence, the carbon products which are obtained through the modified traditional process confirm the purity and can be extended for the production of much needed carbon nanotubes. The traditionally prepared carbon nanoparticles can be primarily used in medical and engineering applications. These are potential secondary reinforcing elements to fabricate fibre reinforced plastic nanocomposites to enhance physical and technological properties. The fibre laminated carbon nanocomposites are effectively being used in aircrafts, space vehicles, automotive structures, ship buildings, and submarines.

\section{Experimental Details}

2.1. Modified Traditional Process Setup. Basically the traditional process is just burning any oil in a lamp and collecting the carbon black [11] from the tip of the flame. This type process is followed since Vedic age. Unlike this, the modified traditional process (MTP) setup uses traditional earthen lamp with central flame tube and stainless steel combustion chamber having the provision to heat up externally. The competitive advantages of the process technique are to produce pure carbon nanomaterials at low cost when compared to any other novel methods.

The process shown in Figure 1 comprises the traditional earthen lamp with oil pot of $100 \mathrm{~mL}$ capacity and a central flame tube or holder of $5 \mathrm{~mm}$ diameter. The flame tube carries cotton thread which can be raised or lowered by a knob with one end immersed in the oil pot while the other end holds the flame. The flame geometry can be adjusted by knob. Controlled combustion is ensured by a stainless steel combustion chamber of $125 \mathrm{~mm}$ length and $90 \mathrm{~mm}$ diameter with circumferential holes. The collector 
TABLE 1: CNCP (Carbon nanocrystal particle) yield (in $\mathrm{Mg}$ ) for $50 \mathrm{~mL}$ oil.

\begin{tabular}{|c|c|c|c|c|}
\hline \multirow{2}{*}{$\begin{array}{l}\text { Time in sec } \\
(50 \mathrm{~mL} \text { oil })\end{array}$} & \multicolumn{2}{|c|}{ MTP1 without external heating yield in Mg } & \multicolumn{2}{|c|}{ MTP2 with external heating yield in Mg } \\
\hline & Animal base (S1) & Mixed veg. base (S2) & Animal base $(\mathrm{S} 1)$ & Mixed veg. base (S2) \\
\hline 900 & 112 & 176 & 269 & 293 \\
\hline 1800 & 147 & 206 & 305 & 327 \\
\hline 2700 & 182 & 231 & 347 & 368 \\
\hline 3600 & 216 & 272 & 419 & 428 \\
\hline 4500 & 253 & 310 & 497 & 521 \\
\hline 5400 & 290 & 338 & 543 & 569 \\
\hline 6300 & 325 & 374 & 576 & 612 \\
\hline 7200 & 359 & 416 & 621 & 649 \\
\hline
\end{tabular}

plate made of ceramic or stainless steel material is used to collect carbon deposits from combustion chamber upper side opening. The combustion chamber can optionally be heated externally (forced convective heating), if required, by an electric resistance coil heater to increase the yield. Hence, the process with above additional set up is named "Modified Traditional Process" indexed as MTP. The entire process should be in a dust-free confined room with absolutely no ambient parametric interferences. The MTP is used to develop two types of samples which are indexed as S1 from animal base and S2 from mixed vegetable base. The samples were prepared by MTP1 without external heating and MTP2 with external heating and compared for their characteristics.

2.2. Preparation, Quantification, and Storage. The modified traditional and catalyst-free process ensures the development of high-purity carbon nanomaterials (CNMs). Samples are prepared from animal base (S1) and vegetable base (S2) oils separately, considering all the ambient parameters. The Figure 1 is the modified traditional process (forced convective heating process) setup for the preparation of samples. Two separate earthen traditional lamps with respective base oils are used. The traditional earthen lamp is first made to burn with respective base oil inside the combustion chamber and externally heated. Due to this forced convective heating, the temperature of the surrounding outer zone of the flame inside the combustion chamber is augmented and maintained. The temperature can be controlled as per the yield requirements [21]. The combustion chamber temperature is compulsorily augmented or maintained during any adverse climatic condition.

The flame geometry is adjusted to be flat and spreading over a collector plate with the help of control knob for high yielding. The surrounding zone of the flame inside the combustion chamber will have the temperature around $300^{\circ} \mathrm{C}$ in case of MTP1 without external heating whereas around $600^{\circ} \mathrm{C}$ for MTP2 with external heating. The surrounding zone temperature of the flame inside the combustion chamber is almost doubled because of the external heating by electric heater. This additional heating increases the total carbon yield to significant percentage. The carbon will start depositing over the surface of the collector plate. Both the samples are collected separately from the collector plate and stored in air tight small plastic containers (Figure 1) for further purification process.

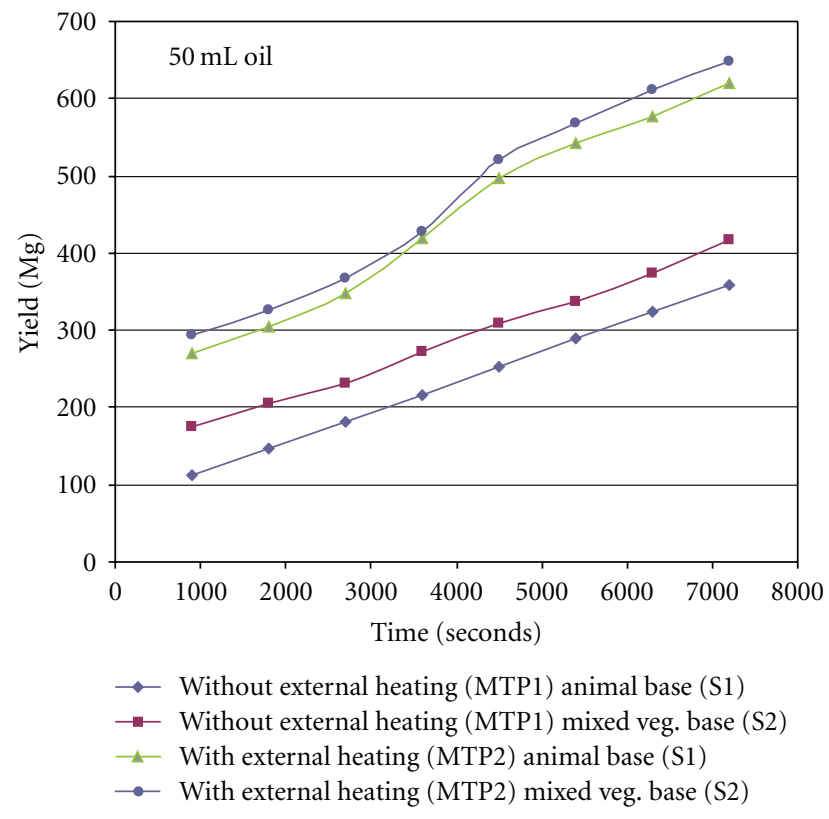

FIgure 2: Time versus CNCP yield in $\mathrm{Mg}$ for $50 \mathrm{~mL}$ oil.

Table 1 shows the observations on weight gain in $\mathrm{Mg}$ during MTP1 (without external heating) and MTP2 (with external heating) processes. The yield at equal intervals of time for $50 \mathrm{~mL}$ of animal (S1) and vegetable (S2) base oils is tabulated. It can be noted clearly that the average weight gain of sample S2 is $22 \%$ for MTP1 and 7\% for MTP2 more than that of the sample S1 over the same period of time.

Figure 2 represents the comparative data for weight gain of the samples over a constant period of time for $50 \mathrm{~mL}$ animal and vegetable base oil through MTP1 and MTP2. It can be revealed from the result that the S2 deposits faster, whereas in the case of $S 1$ the deposition is slower for the same time. This is probably due to fat content of the base oil chosen for the analysis. The quantification is based as it on the ambient conditions, flame geometry, the depositing time, and quantity of base (vegetable/animal) oil in the lamp pot. The collected samples are stored in an air-tight plastic container and kept in a cool and dust-free storage for further purification process. 
TABLE 2: CNCP yield (in Mg) for $100 \mathrm{~mL}$ oil.

\begin{tabular}{lcccc}
\hline $\begin{array}{l}\text { Time in sec } \\
(100 \mathrm{~mL} \text { oil })\end{array}$ & \multicolumn{2}{c}{$\begin{array}{c}\text { MTP1 without external heating yield in Mg } \\
\text { Animal base (S1) }\end{array}$} & Mixed veg. base (S2) & \multicolumn{2}{c}{ MTP2 with external heating yield in Mg } \\
Animal base (S1) & Mixed veg. base (S2) \\
\hline 1800 & 256 & 342 & 426 & 456 \\
3600 & 287 & 381 & 481 & 509 \\
5400 & 315 & 405 & 521 & 543 \\
7200 & 348 & 442 & 589 & 611 \\
9000 & 381 & 486 & 692 & 732 \\
10800 & 425 & 513 & 764 & 784 \\
12600 & 467 & 543 & 827 & 859 \\
14400 & 506 & 568 & & \\
\hline
\end{tabular}

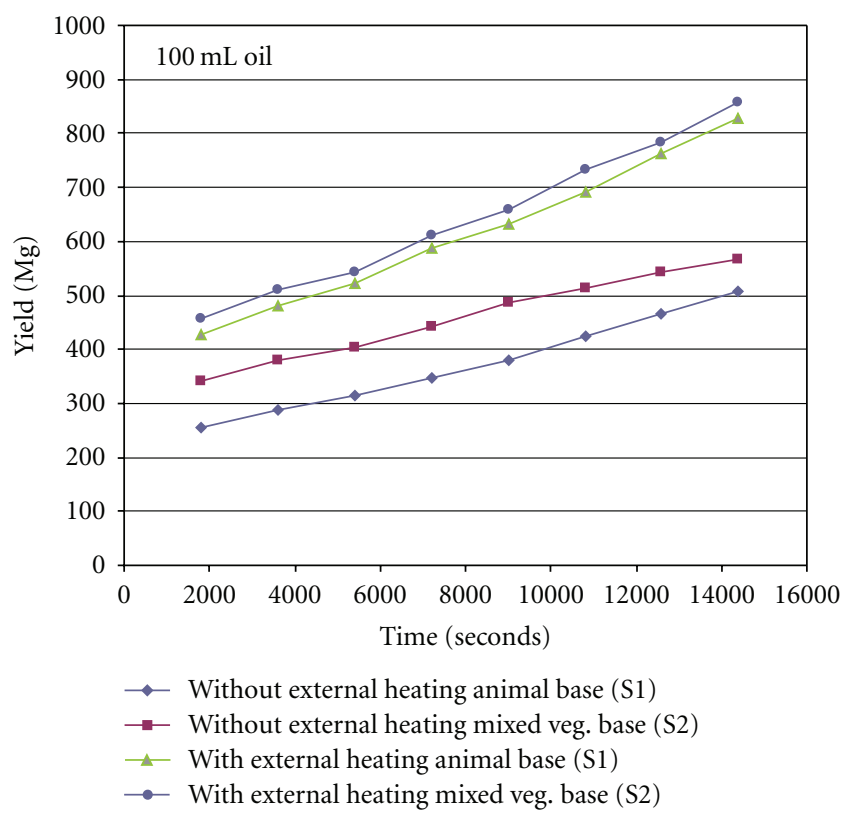

Figure 3: Time versus CNCP yield in $\mathrm{Mg}$ for $100 \mathrm{~mL}$ oil for both the process.

Table 2 shows the observations on weight gain in $\mathrm{Mg}$ during MTP1 and MTP2. The yield at equal intervals of time for $100 \mathrm{~mL}$ of animal (S1) and vegetable (S2) base oils is tabulated. It can be noted clearly that the average weight gain of sample S2 is 27\% for MTP1 and 5\% for MTP2 more than that of the sample S1 over the same period of time.

Figure 3 represents the comparative data for weight gain of the samples over a constant period of time for $100 \mathrm{~mL}$ animal and vegetable base oils, prepared through MTP1 and MTP2. It can be revealed from the result that the S2 deposits more than that of S1 for the same time in both cases.

The percentage gain in yield between the samples is much less, and this is probably due to basic characteristic properties of the base oil chosen for the analysis. The quantification is based on the ambient conditions, flame geometry, the depositing time, and quantity of base (vegetable/animal) oil in the lamp pot.

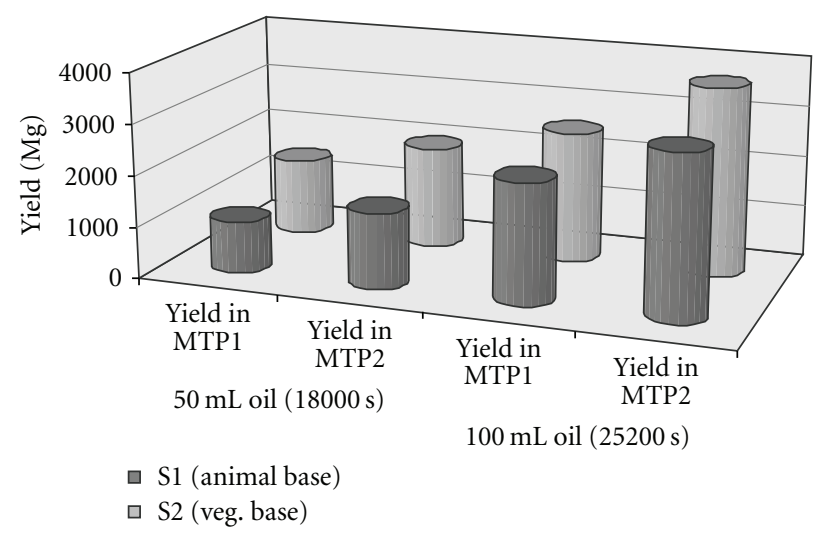

FIgURE 4: Comparative graph for total yield for $50 \mathrm{~mL}$ and $100 \mathrm{~mL}$ for both MTP1 \& MTP2.

The observations in the Table 3 show the comparisons of the total yield of samples for complete burn out time, $18000 \mathrm{sec}$ for $50 \mathrm{mLand} 25200 \mathrm{sec}$ for $100 \mathrm{mLanimal}$ (S1) and (S2) vegetable base oils. The comparisons are made between total yields in MTP1 and MTP2. Product yielding in $18000 \mathrm{sec}$ during the MTP1 and MTP2 is increased about $47 \%$ and $35 \%$, respectively for the sample S2. But the yield for $25200 \mathrm{sec}$ for the same sample S2 during both MTP1 and MTP2 the \% wt. gain is limited to $9 \%$ and $16 \%$, respectively.

Figure 4 shows the comparative results for both S1 \& S2 samples for different quantities of oil for complete burn out time. Observations from both the processes are used to know the gain in weight of total yield for both samples. The percentage weight gain of yield in MTP2 is shown in Figure 5 for both S1 and S2 for different quantities of animal and vegetable base oils. This considerable improvement in yield shows that the MTP is superior when comparing to any other novel method used to produce the carbon nanomaterials.

The main features of the MTP are

(i) it is a simple and low cost process which can be extended to village cottage industries for mass production of carbon nanomaterials;

(ii) the raw materials and the setup components have made the deposition kinetics simple and easy without compromising the quality; 
TABLE 3: Comparisons of product total yield between MTP1 and MTP2.

\begin{tabular}{|c|c|c|c|c|c|c|}
\hline \multirow[b]{2}{*}{$\begin{array}{l}\text { Sample } \\
\text { code }\end{array}$} & \multicolumn{3}{|c|}{$50 \mathrm{~mL}$ oil $(18000 \mathrm{sec})$} & \multicolumn{3}{|c|}{$100 \mathrm{~mL}$ oil $(25200 \mathrm{sec})$} \\
\hline & $\begin{array}{l}\text { Yield in } \\
\text { MTP1 }\end{array}$ & $\begin{array}{l}\text { Yield in } \\
\text { MTP2 }\end{array}$ & $\begin{array}{c}\% \text { age } \\
\text { increase of } \\
\text { yield }\end{array}$ & $\begin{array}{l}\text { Yield in } \\
\text { MTP1 }\end{array}$ & $\begin{array}{l}\text { Yield in } \\
\text { MTP2 }\end{array}$ & $\begin{array}{c}\% \text { age } \\
\text { increase of } \\
\text { yield }\end{array}$ \\
\hline S1 & 1012 & 1476 & 45 & 2344 & 3172 & 35 \\
\hline S2 & 1489 & 1994 & 34 & 2557 & 3679 & 44 \\
\hline$\%$ age & 47 & 35 & & 9 & 16 & \\
\hline
\end{tabular}

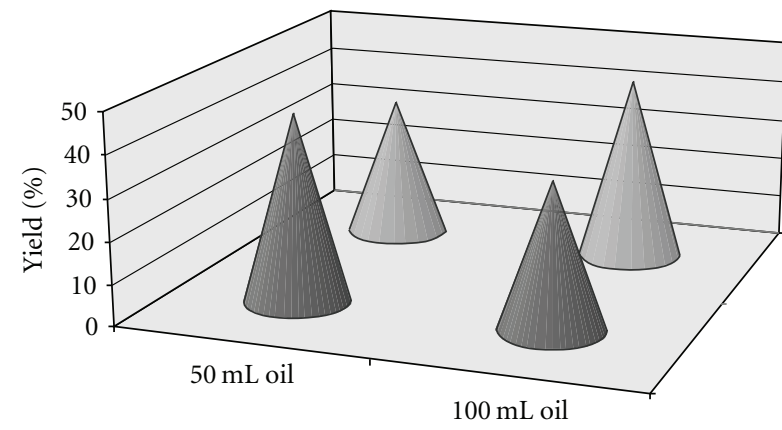

Increase of yield MTP2 (\%)

- S1 (animal base)

․ S2 (veg. base)

Figure 5: Comparative graph for \% wt. gain in the yield for both the quantity of oil in MTP2.

(iii) collected product is entirely free from impurities such as industrial and catalytic inclusions;

(iv) the yield can be maximised by the external heating of combustion chamber whenever required, but compulsorily during cold, rainy and damp seasons.

2.3. Purification Process. The developed samples are purified through a very simple process. It includes the soaking of samples in ethanol for $2 \mathrm{hrs}$ and then washed by the distilled water. The resulted samples were dried in the open furnace for about 10 to $20 \mathrm{~min}$ at about $200-300^{\circ} \mathrm{C}$. The purified samples are then stored in a dust-free storage for further investigations.

2.4. Characterisation Samples. The morphology and structural geometry of the developed samples (S1 \& S2) were deeply investigated using sophisticated analytical instruments. The HRSEM and TEM were employed to investigate the samples at low and high magnifications for the morphology and geometry. The particles analysis was carried out by XRD, EDAX, Raman spectroscope, and TGA. The XRD result was collected over $2 \theta$ range of $5-90^{\circ}$. The laser excitation wave length was $1.5406^{\circ} \mathrm{A}$, and the power was $1.93 \mathrm{~mW}$. The thermal stability of the samples was checked by the Perkin Elmer Pyris 1TGA thermo balance. The samples were heated in $\mathrm{N}_{2}$ environment from $30-900^{\circ} \mathrm{C}$ at $10^{\circ} \mathrm{C} / \mathrm{min}$. Raman spectroscope was used to find the pattern of spectrum and Raman's shift peaks in the curves to confirm the carbonius particles.

\section{Results and Discussions}

3.1. Microscopic Investigations. A simple purification process made the products free from all impurities. The particle analysis through EDAX in Figure 6 for the purified samples S1 \& S2 confirms the presence of only carbon with $100 \%$ purity. When we compare Figure 6 S1 \& S2, it is very clear to say that $\mathrm{S} 1$ took lesser counts ( $7.8 \mathrm{~K}$ counts) to attain the peak earlier to S2 which took $11.0 \mathrm{~K}$ counts for the same energy of $0.25 \mathrm{KeV}$. The sample S2 observed for more counts then also no traces of impurities found in the sample.

The SEM images in Figures 7(a) and 7(d) are representing the low magnified samples S1 and S2, respectively, with size $<200 \mathrm{~nm}$. The purified samples $S 1$ (Figures $7($ b) and $7(\mathrm{c}))$ \& S2 (Figures 7(e) and 7(f)) composed of uniform spherical, ellipsoidal, and short tubular-like nanoparticles with average dimension ranging from 8 to $47 \mathrm{~nm}$ at different magnifications. Further, the SEM images in Figures 7(b) and 7 (c) confirm the nature, uniformity in size, and regular and irregular shapes of the purified sample $S 1$ which is developed out of animal base. Similarly, the sample S2 prepared from veg. base (Figures $8(\mathrm{e})$ and $8(\mathrm{f})$ ) contains pure nanoparticles with uniformity in particle size and also with regular and irregular shapes. The difference between S1 and S2 is the weight gain in total yield over a period of time. In all images, there is no sign of any impurities and only pure carbon nanoparticles are observed.

The review of exclusive TEM study [22, 23] gave a good idea to explain the structure of samples S1 \& S2. The TEM experimental investigations of the samples $\mathrm{S} 1$ (Figures $8(\mathrm{a})$, $8(\mathrm{~b})$, and $8(\mathrm{c}))$ and sample S2 Figures 8(d), 8(e), and 8(f) revealed that the range in dimension of the nanoparticles is varying from 6 to $45 \mathrm{~nm}$. The lengthy and high aspect ratio tubular structures are visible in Figure 8(a). The morphology of the carbon nanomaterials from the MTP shows the thin MWCNT with few defects. The central transparency and hemispherical end in Figure 8(c) confirm the closed end tubular structure with thin multi-walled configuration. Moreover, in the Figure 8(b) the thin tubular nanostructures along with the presence of minimal junctions and bends are presented. The most interesting aspect in the TEM images of the sample S2, Figures $8(\mathrm{~d}), 8(\mathrm{e})$, and $8(\mathrm{f})$, is relatively less complicated but uniform ellipsoidal and spherical structures in a chain formation. The sample S2 contains only the carbon nanocrystals, and these are not perfectly spherical and the particle shape indicates the horizontal and vertical dimensions. The nanocrystals are having surface passivation. But there is likely possibility for the formation of fine tubules. 


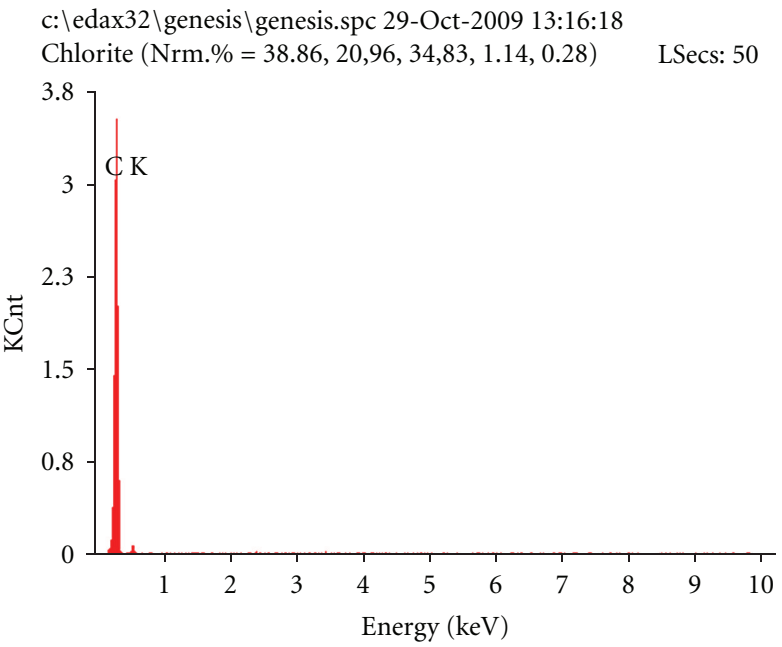

(a) c: \edax32 \genesis \genesis.spc 29-Oct-2009 12:57:48

Chlorite $(\mathrm{Nrm} . \%=38.86,20,96,34,83,1.14,0.28) \quad$ LSecs: 50

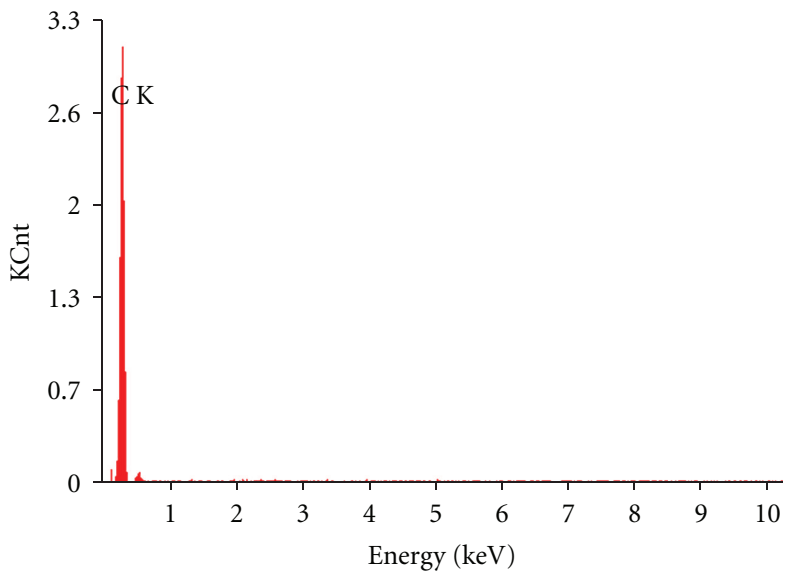

(b)

FIgURE 6: EDAX Data for sample S1 for animal base and S2 for Vegetable base.

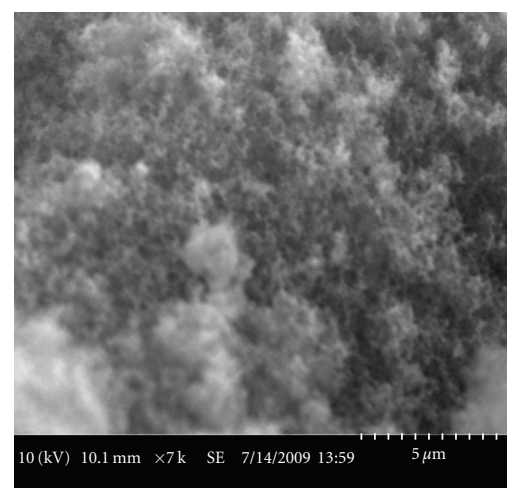

(a)

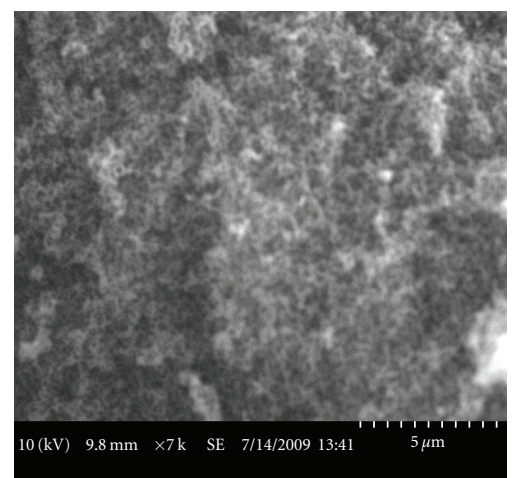

(d)

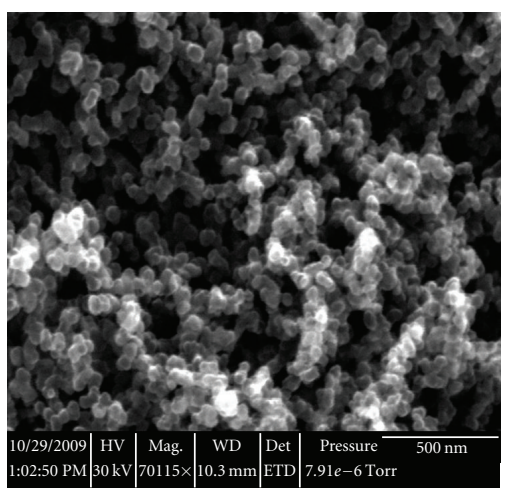

(b)

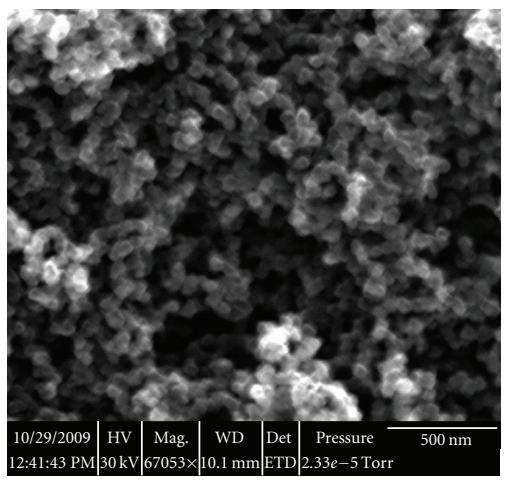

(e)

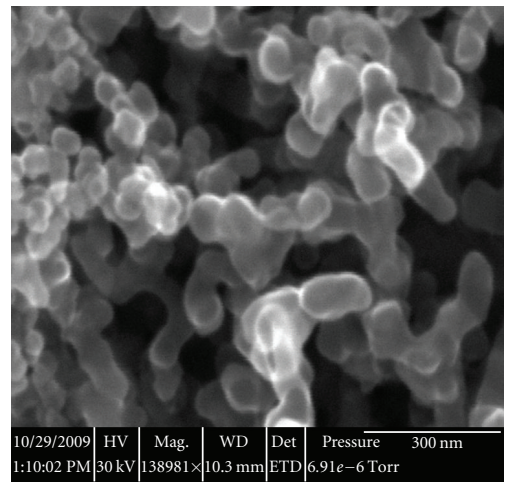

(c)

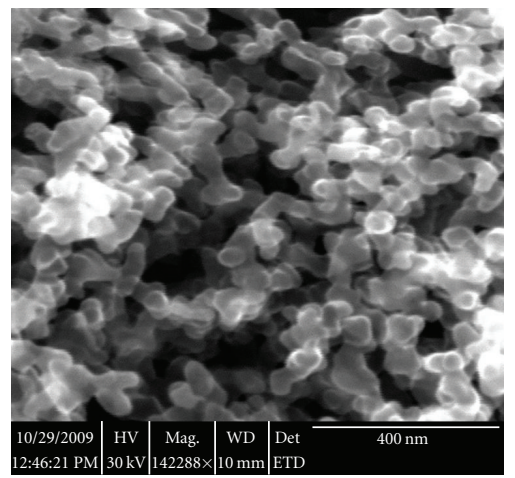

(f)

Figure 7: SEM images (a, b, and c) for sample S1 and (d, e, and f) for sample S2 obtained by using animal and veg. base oils.

The deep investigation has revealed that nanostructures derived out of MTP samples (S1 \& S2) are pure and have fewer defects. The TEM images provide very limited and local information on the samples.

3.2. Thermogravimetric Analysis. The thermal stability and combustion capability of the traditionally prepared samples S1 \& S2 were analysed through TGA thermo balance setup. While the samples were investigated, it was clearly understood that the samples S1 \& S2 were completely burnt as happened with commercial high-purity CNT [24]. Normally, the thin multi-walled carbon nanomaterials the combustion takes place at $550^{\circ} \mathrm{C}$, but the amorphous carbon burns at $300^{\circ} \mathrm{C}$ itself. The analysis confirmed that 


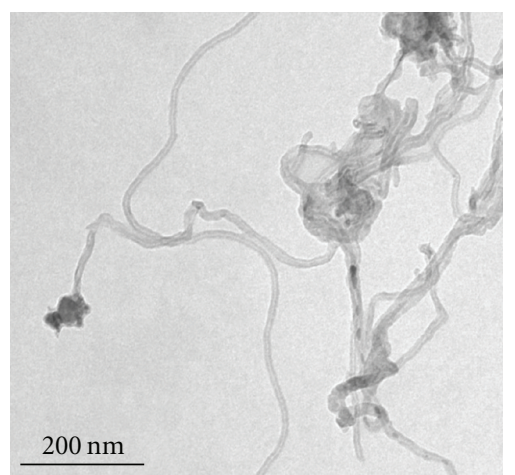

(a)

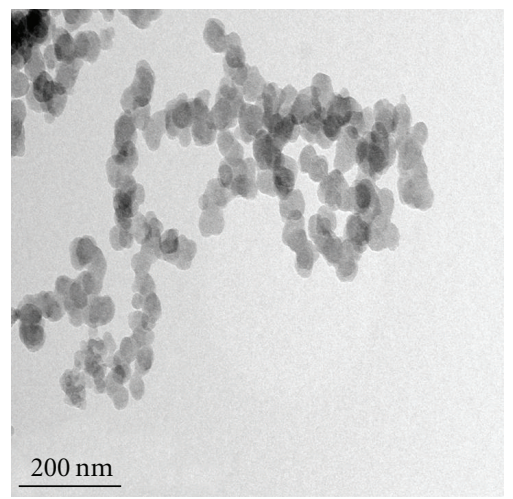

(d)

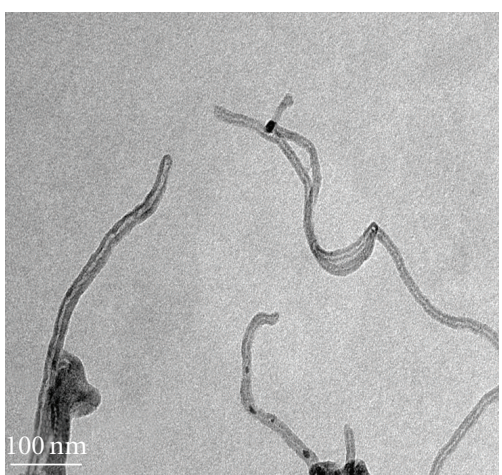

(b)

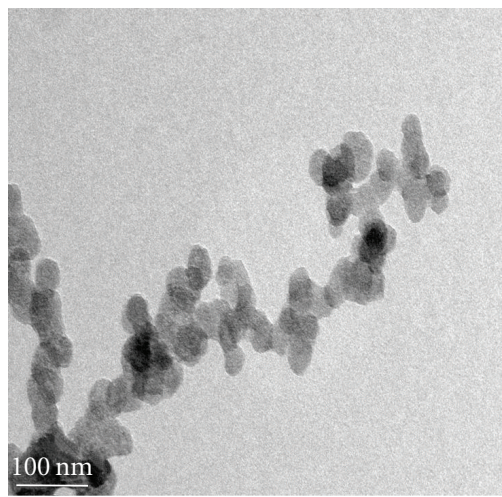

(e)

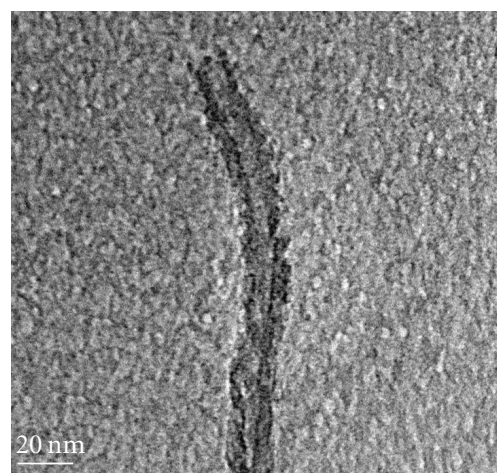

(c)

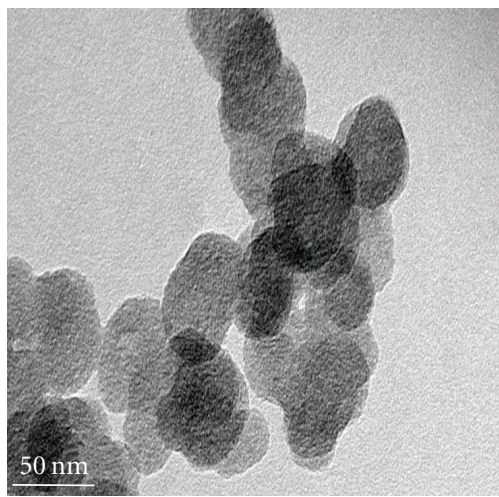

(f)

Figure 8: TEM images ( $a, b$, and c) for sample S1 and images ( $d, e$, and f) for sample S2 obtained by using animal and veg. base oils.

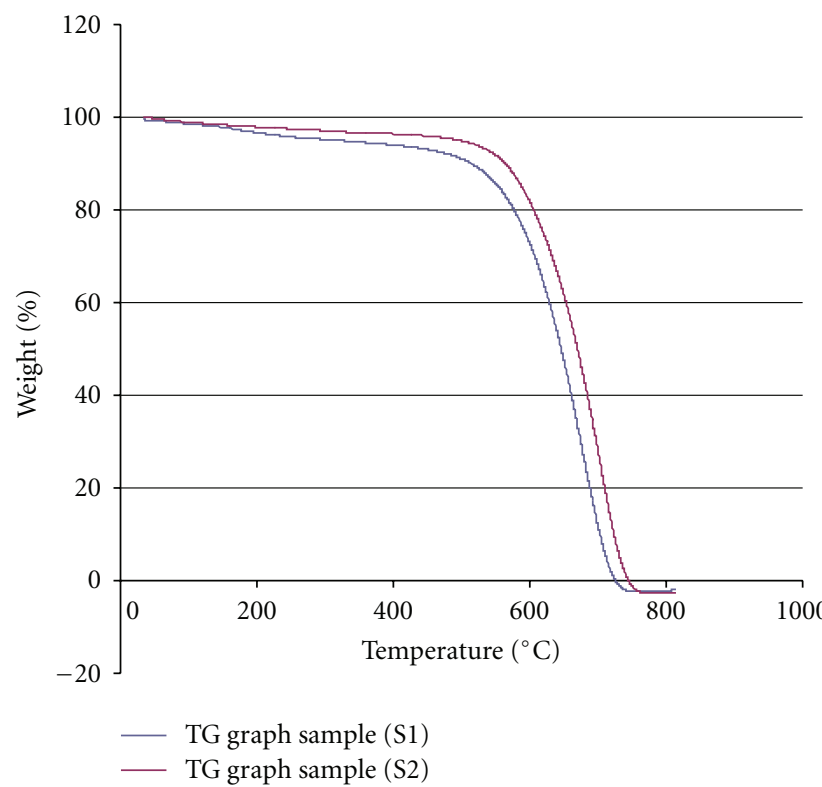

(a)

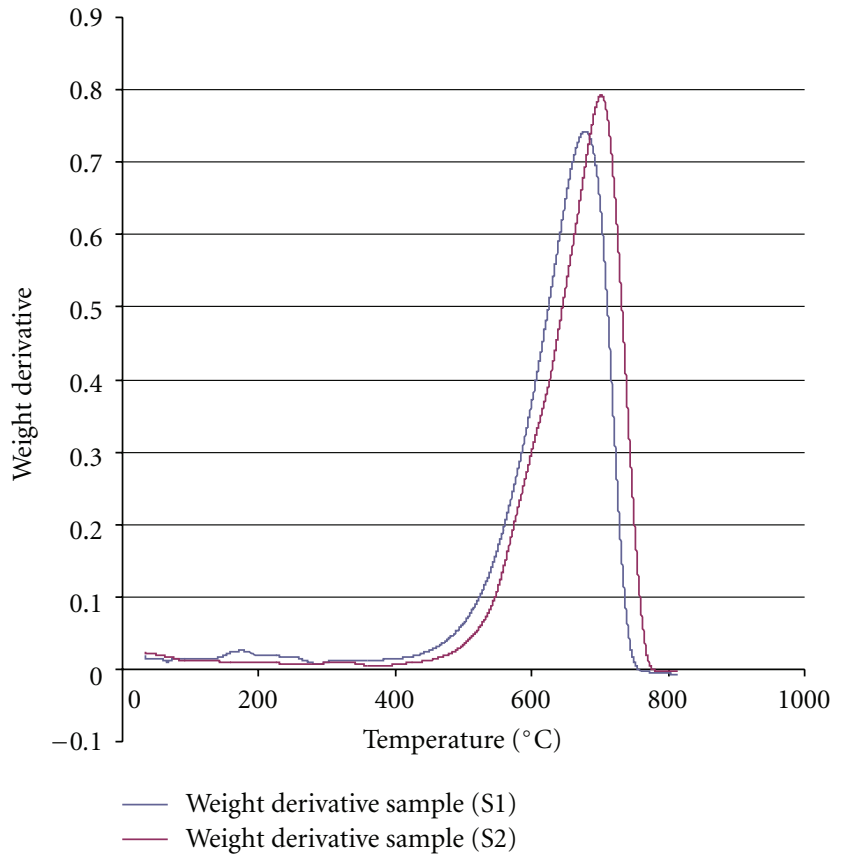

(b)

FIGURE 9: TGA analysis for prepared samples (a) TG curve and (b) differential TG curve. 


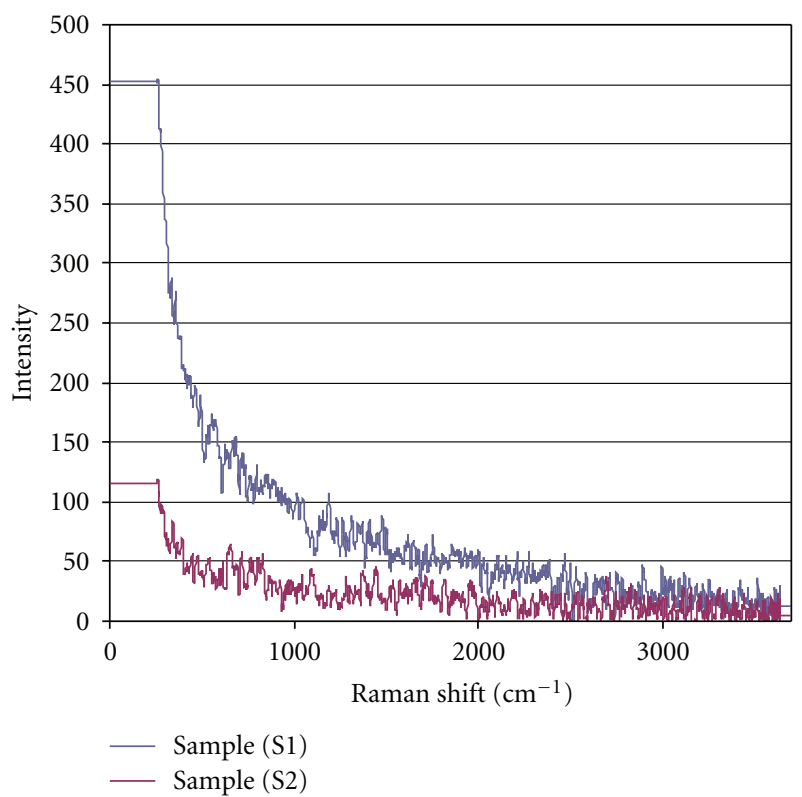

(a)

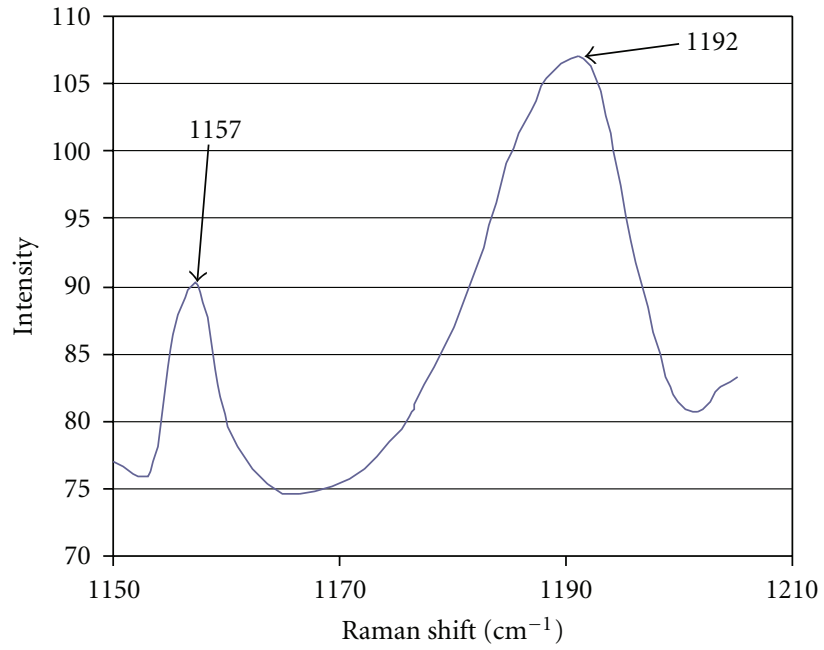

(b)

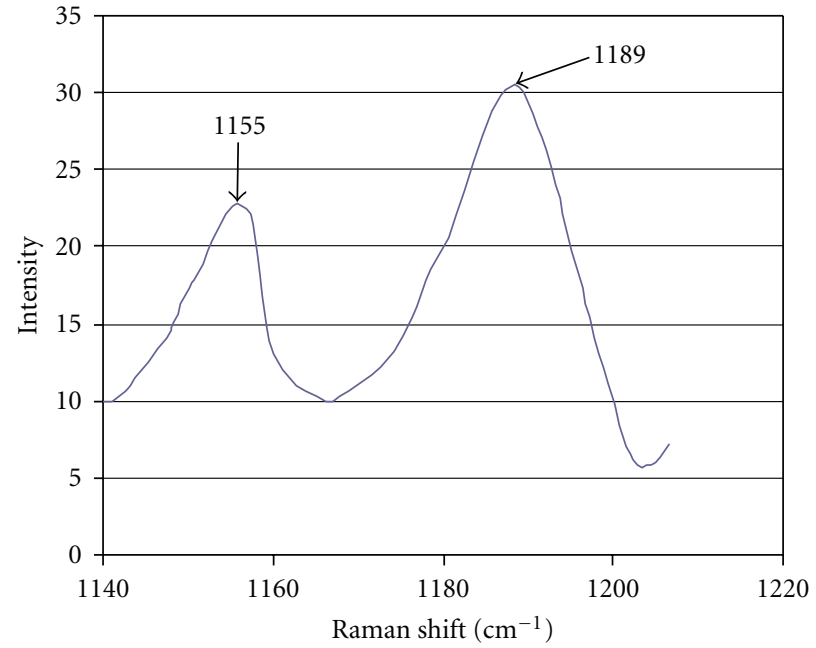

(c)

Figure 10: Raman's full range Spectra for S1 sample and S2 sample (a), whereas (b) and (c) are the graph for a specific range of Raman's shift from $1140-1200 \mathrm{~cm}^{-1}$.

the samples contained $100 \%$ carbon content with almost no traces of impurities. The MTP samples had $0.5 \%$ to $1.5 \%$ weight loss at $100^{\circ} \mathrm{C}$ due to the evaporation of moisture content. But the degradation started at around $570^{\circ} \mathrm{C}$ for sample S1 and $600^{\circ} \mathrm{C}$ for sample S2 in TG curve (Figure 9(a)). The deferential TG curve is shown for the prepared samples S1 \& S2 in Figure 9(b).

3.3. Raman Spectroscopic Analysis. The Raman spectroscopic analysis of MTP samples was carried out under normal ambient conditions. The Raman spectrum recorded for the full range for the samples S1 (Figure 10(a)) and S2 (Figure 10(b)) with nanocrystals provided the evidence for graphitic shells. The specific spectral range between
$1100 \mathrm{~cm}^{-1}$ and $1200 \mathrm{~cm}^{-1}$ had been chosen for the samples S1 \& S2 to recognise some prominent peaks and provides valuable information on the structure of carbon materials. The animal base sample $S 1$ and vegetable base sample S2 have similar Raman characteristics.

3.4. XRD Analysis. The MTP samples, S1 \& S2, were confirmed for their graphitic nature by XRD technique represented by Figure 11(a) for S1 and Figure 11(b) for S2. The high and low intensity peaks were obtained in both the cases, that is, S1 \& S2 samples at $25^{\circ}$ and $44^{\circ}$. This analysis reveals the presence of pure graphitic structures. The MTP did not affect the crystallinity and structural stability of the carbon nanomaterials. 


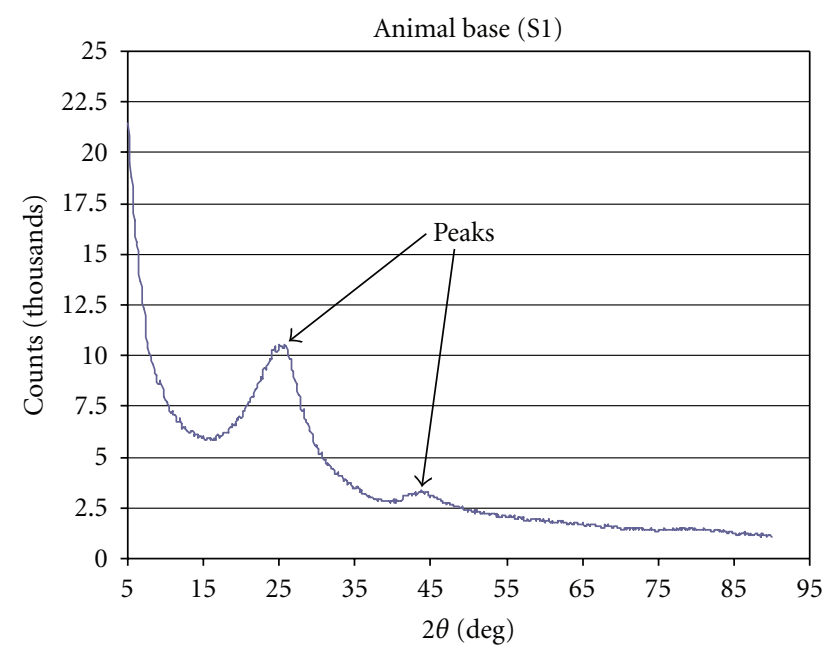

(a)

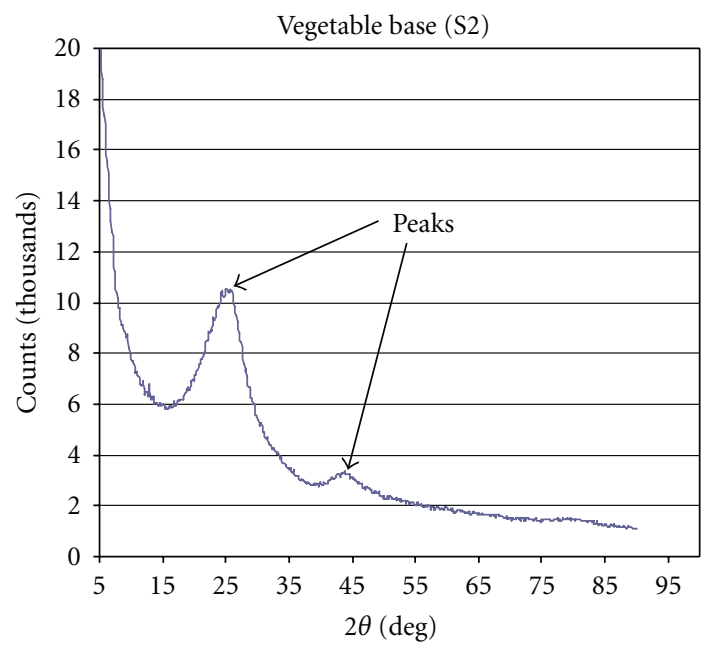

(b)

FIGURE 11: XRD Graphical representations of prepared samples for animal base (a) and veg. base (b).

\section{Conclusion}

The Modified Traditional preparation kinetics and characteristics of the obtained samples were investigated. The MTP was made possible for making pure, high yield, and nontoxic carbon nanomaterials. The results show the considerable increase in the rate of deposition in MTP2. The weight percentage increase is $45 \%$ for S1 yield, and it is 34\% for S2 yield for $18000 \mathrm{sec}$. The purity of samples approaches $100 \%$ as the process is slow and steady, traditional, and nontoxic. Hence, the samples prepared through this process are considered to be superior in its category. Both the samples were characterised by using variety of Sophisticated Instrument techniques. Once the dispersibility of traditionally prepared CNMs is ensured, the particles can used as filler reinforcements to fabricate carbon nanolaminated composite structures which are potentially very much used in aerospace applications. This task will be the future work.
The mass production of the CNMs can be extended to the villages as a cottage small-scale industry which will improve the revenue of the country.

\section{Acknowledgments}

The author P. S. Venkatanarayanan would like to thank Dr. Murthy Professor IITM, Chennai, India for his total encouragement and support throughout the project especially during the characterisation of the prepared samples. The author extended his hearty thanks to Mr. Rajendran from IITM, Chennai, India for their valuable support during the testing of samples. But it is very important for him to say his sincere thanks to all those helped him directly or indirectly to conclude this project successfully.

\section{References}

[1] S. J. Harris and A. M. Weiner, "Chemical kinetics of soot particle growth," Annual Review of Physical Chemistry, vol. 36, pp. 31-52, 1985.

[2] M. Kluüppel, A. Schroüder, and G. Heinrich, "Carbon Black," in Physical Properties of Polymers Handbook, Chemistry and Materials Science, Chapapter 31, pp. 539-550, Springer, Berlin, Germany, 2007.

[3] J. Han, X. Zhang, W. Guo, and C. Wu, "Effect of modified carbon black on the filler-elastomer interaction and dynamic mechanical properties of SBR vulcanizates," Journal of Applied Polymer Science, vol. 100, no. 5, pp. 3707-3712, 2006.

[4] J.-B. Donnet, R. C. Bansal, and M.-J. Wang, Carbon black, Marcel Dekker, New York, NY, USA, 2nd edition, 1993.

[5] B. R. Puri, Chemistry and Physics of Carbon, vol. 6 of P. L. Walker Jr., Ed., Marcel Dekker, New York, NY, USA, 1970.

[6] H. P. Boehm, "Some aspects of the surface chemistry of carbon blacks and other carbons," Carbon, vol. 32, no. 5, pp. 759-769, 1994.

[7] S. Iijima, "Helical microtubules of graphitic carbon," Nature, vol. 354, no. 6348, pp. 56-58, 1991.

[8] R. F. Klie, D. Ciuparu, L. Pfefferle, and Y. Zhu, "Multi-walled carbon nanotubes on amorphous carbon films," Carbon, vol. 42, no. 10, pp. 1953-1957, 2004.

[9] L. E. Murr, J. J. Bang, E. V. Esquivel, P. A. Guerrero, and D. A. Lopez, "Carbon nanotubes, nanocrystal forms, and complex nanoparticle aggregates in common fuel-gas combustion sources and the ambient air," Journal of Nanoparticle Research, vol. 6, no. 2-3, pp. 241-251, 2004.

[10] I. Stamatin, A. Morozan, A. Dumitru et al., "The synthesis of multi-walled carbon nanotubes (MWNTs) by catalytic pyrolysis of the phenol-formaldehyde resins," Physica E, vol. 37, no. 1-2, pp. 44-48, 2007.

[11] P. Dubey, D. Muthukumaran, S. Dash, R. Mukhopadhyay, and S. Sarkar, "Synthesis and characterization of water-soluble carbon nanotubes from mustard soot," Pramana - Journal of Physics, vol. 65, no. 4, pp. 681-697, 2005.

[12] O. Prilutskiy, E. A. Katz, A. I. Shames et al., "Synthesis of carbon nanomaterials by a catalytic disproportionation of carbon monoxide," Fullerenes Nanotubes and Carbon Nanostructures, vol. 13, no. S1, pp. 53-66, 2005.

[13] A. Mangione, L. Torrisi, A. Picciotto et al., "Carbon nanocrystals produced by pulsed laser ablation of carbon," Radiation Effects and Defects in Solids, vol. 160, no. 10-12, pp. 655-662, 2005. 
[14] M. Bystrzejewski, M. H. Rummeli, T. Gemming, H. Lange, and A. Huczko, "Catalyst-free synthesis of onion-like carbon nanoparticles," New Carbon Materials, vol. 25, no. 1, pp. 1-8, 2010.

[15] S. R. C. Vivekchand and A. Govindaraj, "A new method of preparing single-walled carbon nanotubes," Proceedings of the Indian Academy of Sciences: Chemical Sciences, vol. 115, no. 56, pp. 509-518, 2003.

[16] L. Gan, H. D. Du, B. H. Li, and F. Y. Kang, "The effect of particle size on the interaction of Pt catalyst particles with a carbon black support," Xinxing Tan Cailiao/ New Carbon Materials, vol. 25, no. 1, pp. 53-59, 2010.

[17] B. C. Yadav and R. Kumar, "Structure, properties and applications of fullerenes," International Journal of Nanotechnology and Applications, vol. 2, no. 1, pp. 15-24, 2008.

[18] J. M. Cowley, R. C. Mani, M. K. Sunkara, M. O’Keeffe, and C. Bonneau, "Structures of carbon nanocrystals," Chemistry of Materials, vol. 16, no. 24, pp. 4905-4911, 2004.

[19] PH. Lambin, J. F. Colomer, L. Henrard, A. Lucas, and V. Meunier, "Structural characterization of carbon nanotubes," Microscopy and Microanalysis, vol. 12, no. 2, pp. 570-571, 2006.

[20] Y. Suda, T. Ono, M. Akazawa, Y. Sakai, J. Tsujino, and N. Homma, "Preparation of carbon nanoparticles by plasmaassisted pulsed laser deposition method-size and binding energy dependence on ambient gas pressure and plasma condition," Thin Solid Films, vol. 415, no. 1-2, pp. 15-20, 2002.

[21] N. Sano, T. Charinpanitkul, T. Kanki, and W. Tanthapanichakoon, "Controlled synthesis of carbon nanoparticles by arc in water method with forced convective jet," Journal of Applied Physics, vol. 96, no. 1, pp. 645-649, 2004.

[22] U. K. Gautam and B. Mukherjee, "A simple synthesis and characterization of CuS nanocrystals," Bulletin of Materials Science, vol. 29, no. 1, pp. 1-5, 2006.

[23] P. J. F. Harris, "Carbon nanotubes and other graphitic structures as contaminants on evaporated carbon films," Journal of Microscopy, vol. 186, no. 1, pp. 88-90, 1997.

[24] Y. Li, E. C. Y. Liu, N. Pickett et al., "Synthesis and characterization of CdS quantum dots in polystyrene microbeads," Journal of Materials Chemistry, vol. 15, no. 12, pp. 1238-1243, 2005. 

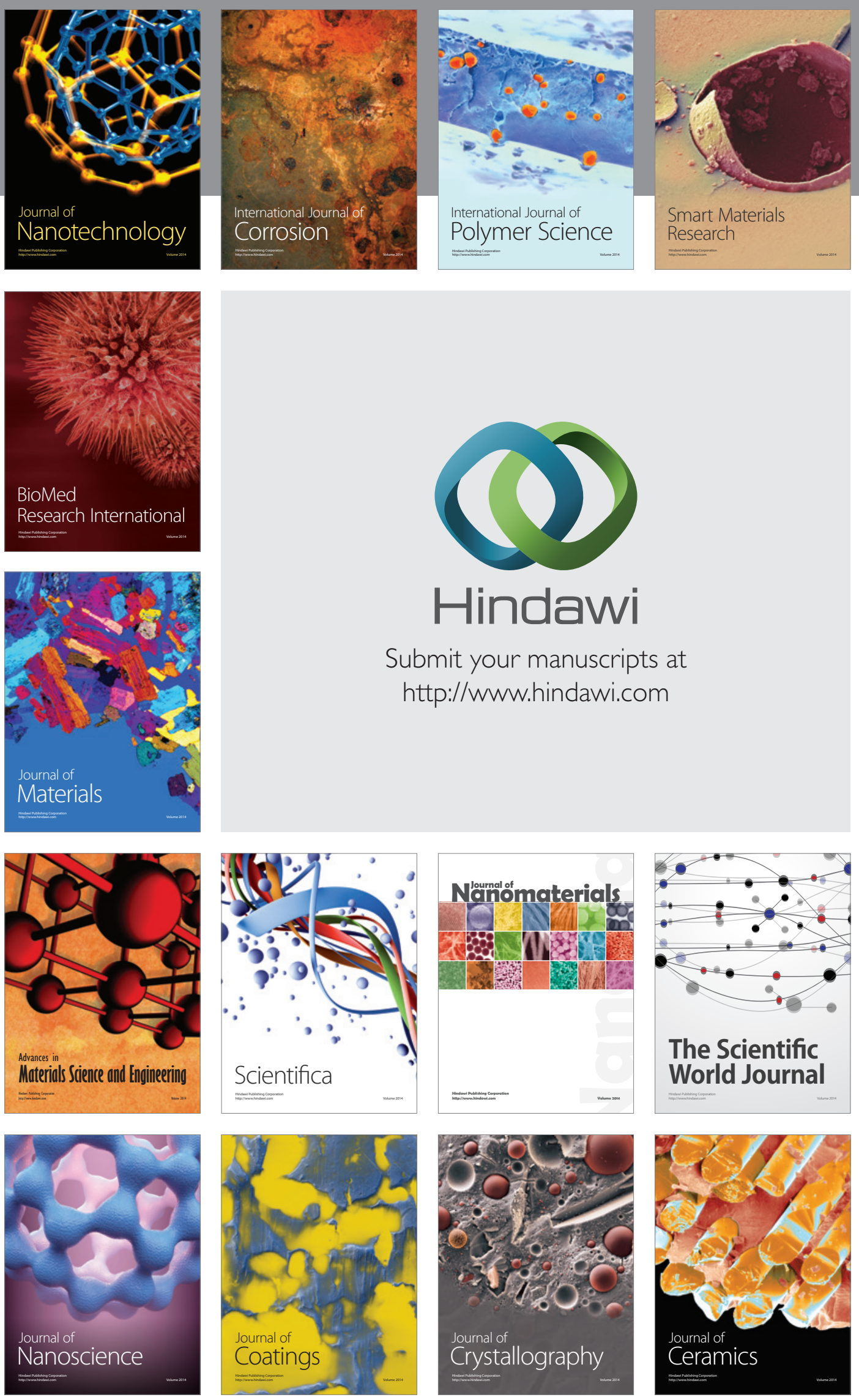

The Scientific World Journal

Submit your manuscripts at

http://www.hindawi.com

\section{World Journal}

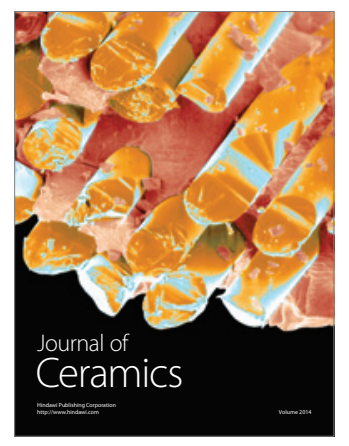

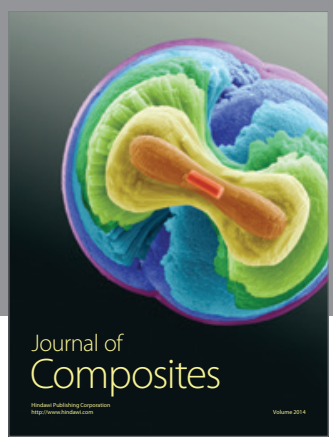
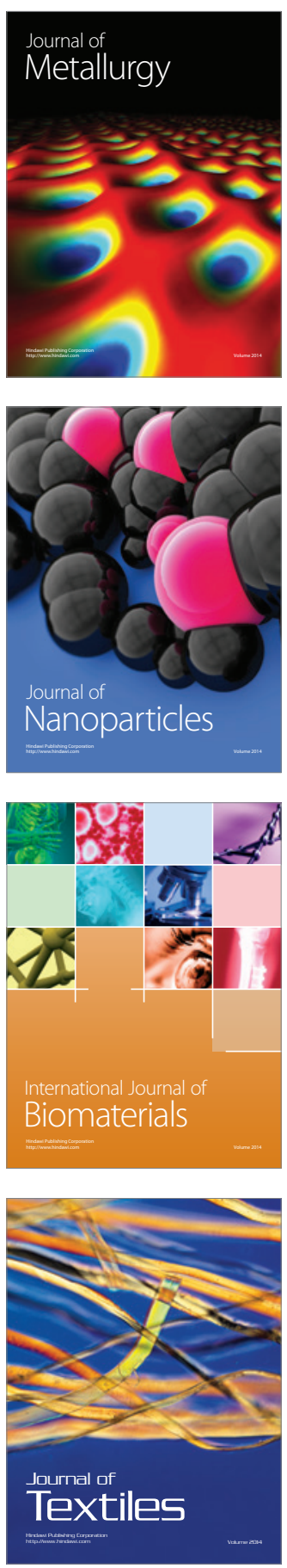